\title{
Learning through inter- and intradisciplinary problem solving: using cognitive apprenticeship to analyse doctor-to-doctor consultation
}

\author{
Christoph Pimmer • Norbert Pachler $\cdot$ Julia Nierle $・$ Urs Genewein
}

Received: 11 September 2011/ Accepted: 11 January 2012/Published online: 1 February 2012

(C) Springer Science+Business Media B.V. 2012

\begin{abstract}
Today's healthcare can be characterised by the increasing importance of specialisation that requires cooperation across disciplines and specialities. In view of the number of educational programmes for interdisciplinary cooperation, surprisingly little is known on how learning arises from interdisciplinary work. In order to analyse the learning and teaching practices of interdisciplinary cooperation, a multiple case study research focused on how consults, i.e., doctor-to-doctor consultations between medical doctors from different disciplines were carried out: semi-structured interviews with doctors of all levels of seniority from two hospital sites in Switzerland were conducted. Starting with a priori constructs based on the 'methods' underpinning cognitive apprenticeship (CA), the transcribed interviews were analysed according to the principles of qualitative content analysis. The research contributes to three debates: (1) socio-cognitive and situated learning, (2) intra- and interdisciplinary learning in clinical settings, and (3), more generally, to cooperation and problem solving. Patient cases, which necessitate the cooperation of doctors in consults across boundaries of clinical specialisms, trigger intra- as well as interdisciplinary learning and offer numerous and varied opportunities for learning by requesting doctors as well as for on-call doctors, in particular those in residence. The relevance of consults for learning can also be verified from the perspective of CA which is commonly used by experts, albeit in varying forms, degrees of frequency and quality, and valued by learners. Through data analysis a model for collaborative problem-solving and help-seeking was developed which shows the interplay of pedagogical 'methods' of CA in informal clinical learning contexts.
\end{abstract}

C. Pimmer $(\bowtie) \cdot$ J. Nierle

University of Applied Sciences Northwestern Switzerland FHNW, Peter Merian-Strasse 86, 4002 Basel, Switzerland

e-mail: Christoph.Pimmer@fhnw.ch

N. Pachler

Institute of Education, University of London, London, UK

U. Genewein

University Hospital, Basel, Switzerland 
Keywords Cognitive apprenticeship - Consultation and referral - Cooperative behavior . Informal learning · Interdisciplinary communication · Learning · Problem-based learning · Problem solving · Workplace learning

\section{Introduction and background}

\section{Specialisation and interdisciplinary cooperation}

Highly specialised health professionals and a need for cooperation are two central characteristics of today's health system: complexities in patient care, technological advances and increasing clinical knowledge are resulting in a great degree of specialisation in health professionals, a greater number of different professions as well as more and more specialties and subspecialties within professions. This, in turn, requires persons with different professional backgrounds to collaborate in order to treat patients effectively (Barr et al. 2005; Hall and Weaver 2001). Consequently, hospitals can be described as a "collection of highly specialised experts" (Becker et al. 2009) functionally organised into departments (Bardram and Bossen 2005). Recently there is much attention paid to processes that focus on the need of customers, in particular patients, across these functional units (Gemmel et al. 2008). In medical literature this perspective is reflected, for example, in the notion of patient-focused care or clinical pathways (Vogel 1993).

Interdisciplinary work in healthcare is, however, not a commonly agreed concept (Lavin et al. 2001). It can include both cooperation between members from the same discipline or profession, for example a team of surgeons and internists, and cooperation between actors with different professional backgrounds such as physiotherapists, surgeons and occupational therapists. Interdisciplinary cooperation involves the solving of a common set of problems where "each member's assessment must take into account the other's contributions to allow for holistic management" (Hall and Weaver 2001).

A typical example for interdisciplinary cooperation of specialised health professionals across organisational units is a 'consult', a doctor-to-doctor consultation. There, a (requesting) doctor involves an on-call doctor from a (sub-)speciality for a patient case that lies outside his/her expertise. Consults are important and common aspects of clinical practice. Studies report, for example, that in emergency departments (ED) the quantity of cases that require consults is about $40 \%$ (Cortazzo et al. 1993); in $7 \%$ of the cases even two consults take place (Woods et al. 2008).

In view of the increasing importance of interdisciplinary work, such as interactions within consults, the main purpose of this research was to explore whether and how interdisciplinary cooperation and problem solving facilitates learning and teaching practices from a socio-cognitive perspective. Accordingly, the research connects to the topics of learning through problem solving, interdisciplinary learning as well as to situated and socio-cognitive perspectives on clinical learning. These topics are introduced and briefly discussed in the following sections:

\section{Learning through (cooperative) problem solving}

In the literature importance is afforded to learning through breakdowns and problem solving in the clinical workplace. In a study on the learning of residents in an ambulatory clinic, breakdowns, i.e., "interruptions in the smooth flow of activity", were identified as 
the most important category because of "their frequency and explanatory power" (Smith et al. 2004). While effective responses to breakdowns were closely associated with learning, a lack of breakdowns rarely resulted in learning or improvement. Similarly, Slotnick conceives the solving of ill-structured problems as the main route of learning for doctors. He considers learning as gaining insights from a multi-stage problem solving process. He distinguishes specific problems that require immediate action from general problems, which are associated with more deliberate learning and planning (1999). In order for learners to be able to broaden their competences through deliberate practice, among other things immediate feedback on their actions is considered to be essential. In the medical context this can mean that more experienced colleagues act as providers of feedback or that less experienced colleagues can compare their actions to those of more experienced peers (Ericsson 2004, 2008). Sargeant et al. (2006), for example, reported that discussions with colleagues about patients were considered fundamental to learning and that in particular residents frequently consulted colleagues in their clinical work: they asked for advice when they deemed their knowledge to be insufficient for treating specific cases. The doctors approached different colleagues according to the particularities of the case and their colleagues' expertise (van de Wiel et al. 2010). In particular during patient encounters doctors tend to ask colleagues for advice instead of studying books or searching the internet (Bennett et al. 2006). Most of the questions raised by sub-specialists focused on problems outside their specialties (Covell et al. 1985). The literature has little to say, however, about how learning takes place and through what forms of social interaction, for example patterns of cooperation, and social practices.

\section{Learning in interdisciplinary settings}

In spite of the increasing importance of interdisciplinary work in healthcare and the potential of collaborative problem solving for competence development in general, little is known about learning associated with interdisciplinary cooperation. The majority of the studies on interdisciplinary cooperation focus on problems in inter-professional teamwork and the effectiveness of educational measures to address these challenges (Carpenter 1995; Lavin et al. 2001; Parsell and Bligh 1998; Sternas et al. 1999). Clinical professionals do not only learn for but also from interprofessional cooperation. There is, however, much less literature that analyses learning in interdisciplinary settings (Hall and Weaver 2001). Some studies indicate, for example, that learning by medical students and doctors was facilitated through interaction with nurses (Dornan et al. 2007; Fagin 1992). While most of the clinical cooperation is dyadic, these forms of cooperation are widely ignored in the interdisciplinary literature (Barr et al. 2005). The focus in the existing literature is placed on educational programmes for interprofessional cooperation in teams, but not on learning from-more loosely framed-intraprofessional cooperation of doctors from different specialties and sub-specialties. The lack of studies that examine interdisciplinary cooperation from a learning perspective can also be confirmed with respect to consults: there are only few studies on consults in general. Most of them are descriptive in nature, lack methodological rigour (Lee et al. 2008) and focus on waiting time and response patterns (Lee et al. 2008; Vosk 1998). Very little is known about learning that is associated with consults. We identified one paper suggesting that the requesting doctors "value brevity and clarity but they also appreciate consultants who make an active effort to share their expertise" (Goldman et al. 1983). Another study indicated the learning value of bedside teaching of on-call doctors within consults (Salerno et al. 2007). 
Situated, socio-cognitive perspectives and cognitive apprenticeship

The understanding of learning in this paper is based on socio-cognitive and situated approaches; they lend themselves particularly well to explaining learning as social interaction in collaborative, complex and unstable systems such as the clinical context (Bleakley 2006). According to theories of situated learning, context in medical education and practice goes well beyond the physical environment. It should be considered as a complex system that evolves over time and consists of interactions between doctors, patients and the setting; the interactions are not predictable and non-linear (Durning et al. 2010). Situated learning can provide a helpful framework to analyse social aspects of learning, in particular to understand cooperation and teamwork in medical practice (Patel et al. 2008). CA can be considered as a situated and socio-cognitive approach to learning. It is rooted "intra-individual cognitive processes" but accounts as well for learning that is situated in problem solving and socio-cultural settings (Kirschner 2006). The main tenets of CA are that learning is actively constructed and situated in order to help learners understand the purposes and meanings of knowledge (Collins et al. 1991). CA intends to make "thinking visible" and is considered an "instructional paradigm" for situated teaching and learning (1991). The approach was developed in the 1980s on the basis of reading, writing and mathematical problem solving (Collins et al. 1987, 1991). Apprenticeship learning is, and has always been, central to medical education (Balmer et al. 2008; Collins et al. 1991; Furmedge 2008). CA, however, combines the traditional apprenticeship approach with more cognitive methods of education (Collins et al. 1991). CA describes six 'methods' of how a teacher can provide students with the "opportunity to observe, engage in, and invent or discover expert strategies in context", namely: modelling, coaching, scaffolding, articulation, reflection and exploration (Collins et al. 1991). These 'methods' of CA have been used in medical and clinical competence development to design learning environments for students (Woolley and Jarvis 2007) as well as to analyse students' learning experiences during longer 'clerkships' (Stalmeijer et al. 2008).

\section{Methods}

Research questions

On the basis of the existing literature, and on the basis of a socio-cognitive and situated understanding of learning, we formulated the following guiding research questions to explore interdisciplinary learning in the context of consults:

RQ1 How do doctors experience consults; who can learn in doctor-to-doctor consultations and to what extent are they considered relevant for competence development?

RQ2 How do the pedagogical methods associated with CA facilitate learning in interdisciplinary cooperation such as consults?

With the first question we intended to explore how doctors experience consultation practices and whether they value them for their own professional learning. Then, in order to analyse how learning occurs in and through consults, we used CA, a common frame that includes different teaching methods. To do so, we chose a qualitative case study approach that allows the understanding of complex phenomena such as interdisciplinary cooperation practices that are tightly coupled within the socio-organizational context of clinical 
environments in which they are situated (Eisenhardt 1989) and to answer questions of whether, how and why (Giacomini and Cook 2000) learning takes place. Case studies were considered valuable and popular in healthcare (Baxter and Jack 2008) as well as in education (Merriam 1998).

\section{Context and sampling}

We chose a multiple case study design to cover potential variations of consultation practices. The research was undertaken in two different hospitals varying in size and scope: one of the largest Swiss university hospitals and a smaller cantonal hospital. Due to the rotation of medical doctors, the participants reported from a much broader range of consultation experiences from hospitals across Switzerland and Germany. We analysed consults between emergency departments (ED) and other (sub-)specialist departments, due to the broad range of specialists with whom emergency doctors cooperate as well as due to the high frequency of requests for consults (Go et al. 1998). In Switzerland and Germany most of the residents spend time in ED even if they do not intend to specialise in emergency medicine. For this reason, many of the interviewed on-call doctors had worked as residents in the ED and, as a consequence, were able to reflect on consults also from this point of view. While the study was part of a longer research program on clinical cooperation, the interviews took place from January to March 2011. In analysing consults between the ED and other specialities in two different sites and in interviewing all roles involved in consults we used typical case sampling (Patton 2002). To do so, we conducted semi-structured interviews with ten participants including five (requesting) doctors from the emergency department and five participants who regularly took the role of on-call doctors. Similarly, half of the participants were from a larger university hospital while the other half worked in the smaller cantonal hospital (Table 1).

Data collection and analysis

The interviews started with open questions about consults. Particular attention was paid to causes, forms of cooperation and processes, roles, technologies in use and frequency. The discussion of themes that emerged during the interviews was explicitly encouraged.

Table 1 Description of sample characteristics

\begin{tabular}{llllll}
\hline & (Intended) speciality/department & Seniority & $\begin{array}{l}\text { Current role } \\
\text { in consults }\end{array}$ & Hospital & Gender \\
\hline 01 & Hand surgery & Resident & On-call & University Hospital & Male \\
02 & Internal and emergency medicine/ED & Attending & Requesting & University Hospital & Male \\
03 & Pathology/ED & Resident & Requesting & Cantonal Hospital & Male \\
04 & Internal medicine/ED & Resident & Requesting & Cantonal Hospital & Female \\
05 & Orthopaedics, hand surgery & Attending & On-call & University Hospital & Male \\
06 & Geriatric medicine/ED & Resident & Requesting & University Hospital & Female \\
07 & General and hand surgery & Resident & On-call & Cantonal Hospital & Male \\
08 & Plastic and general surgery & Resident & On-call & University Hospital & Male \\
09 & Internal medicine/ED & Resident & Requesting & Cantonal Hospital & Male \\
10 & General surgery & Resident & On-call & University Hospital & Female \\
\hline
\end{tabular}


Similarly to van de Wiel et al. (2010) we discussed the doctors' perspectives on learning in consults in the second part of the interview. (For the interview guide see the "Appendix".) The reason for this approach was to avoid bias in relation to what doctors considered relevant for learning as well as in order to enable an analysis from the perspective of situated learning. All the interviews were audio recorded and transcribed verbatim. The data analysis was conducted by four researchers with different backgrounds: three educational researchers with respective expertise in medicine, applied linguistics, social sciences, technology-enhanced learning and one researcher from the political sciences. Similar to Smith et al. (2004) we found it helpful that one researcher (doctor with background in medical education) was an 'insider' in the two clinics and was, therefore, able to interpret findings from the socio-cultural and political context of the research sites. The others acted as 'outsiders' and were, accordingly, able to question things that otherwise might have been taken for granted. The interviews were analysed according to the principles of qualitative content analysis (Mayring 2004) using the CA methods to guide the coding. In a first round the four researchers analysed and discussed $50 \%$ of the data and identified emerging themes. Then two of the researchers coded all the data using NViVo. Similar to Smith et al. (2004) inductive and deductive coding methods were combined: while the coders inductively identified relevant themes to answer RQ1, they took the teaching methods of CA as a priori constructs for a deductive category application. The coded material and the interpretation was, finally, reviewed and critically discussed by all authors until consensus was reached. ${ }^{1}$

\section{Ethical considerations}

Ethical advice was sought from the regional ethical review board. The committee decided that on the basis of the research proposal no further ethical approval was required so long as informed consent was obtained. Anonymity of participants was ensured and written consent was obtained from all interviewees before the interview; and all of them allowed conversations to be audio-taped.

\section{Results}

How doctors experienced and valued consults for learning

RQ1 How do doctors experience consults; who can learn in doctor-to-doctor consultations and to what extent are they considered relevant for competence development?

\section{Consults: processes and characteristics}

Before we discuss learning-related findings, we briefly describe how doctors perceive consultation processes and practices. According to our analysis no such thing as a "standard" consult exists; instead there were a lot of process variations. However, we identified typical patterns that appear to transcend individual hospitals and situations. Consultations were initialised in situations marked by insecurity. In many cases a resident identified a problem for which s/he required expert support from another (sub-)specialism.

\footnotetext{
${ }^{1}$ One author (NP) translated the interview data from German into English.
} 
- I carry out a consult when I don't know how to proceed and/or when I'm not sure exactly what to do. $[08]^{2}$

Upon approval from the attending doctor, s/he requested the on-call doctor in the specialist department, who was usually a resident. While minor questions were solved on the phone, many cases necessitated on-site examination by the on-call doctor. In case of any further questions residents on-call involved the attendings on-call, acting, in turn, as consultees. Again, the involvement of the more experienced doctor tended to take place in a situation marked by insecurity:

- It is possible that in the case of insecurities he confers with the senior physician; otherwise he decides for himself. [03]

In any case they needed to inform their attendings about consults they perform. Once the examinations had been completed, a debriefing took place. Some cases required further post-processing such as referrals, relocations or further medical appointments. Some cases were presented and discussed in team meetings and reports. While a consult was able to involve different roles, the interaction patterns were mostly dyadic. In small hospitals, however, requesting doctors had fewer specialists at hand and often exclusively referred to their attendings.

Because [in a small hospital] few specialists are available for consultation [...]. That means that in internal medicine as well as in surgery other disciplines have to be covered as well. [09].

In general, a consult was considered a common and frequent practice: the doctors in the ED indicated that they needed to consult specialists on a daily basis, with varying frequency depending on the patient census. A resident in the emergency department explained that he requested consults from different specialisms or sub-specialisms on a daily basis, "four to five times on average" [03]. He illustrated this by providing the following example:

- For example, today we had the hand surgeon here four times. The orthopaedist, on the other hand, was not needed. Instead, the urologists were also here twice during my shift today. Those are the most frequent. [03]

It was reported that most of the residents who worked in clinical settings have to take the role of on-call doctors on a regular basis. As such, they worked on several consults daily, up to 10 , as a doctor from the hand surgery department stated. Another resident with a background in general surgery confirmed the high number of consults. "Yes, numerous per day. [...] There are many cases daily." [10]. The most active roles in consults were played by residents, who worked in hospitals as part of their specialist training; this makes the need for an analysis of doctor-to-doctor consultation practices from a learning perspective even more significant.

\section{Perceived learning value of consults}

The vast majority of participants, in particular residents, identified learning in doctor-todoctor consultation as central to, and as one of the most relevant activities for their

\footnotetext{
2 The number after each quotation links to the respective study participant. For example [01] refers to participant with the number 01 in the Table 1 "Description of sample characteristics".
} 
competence development. The solving of complex problems with more experienced doctors, an inherent characteristic of consults, was regarded as the most important learning activity.

- I'd say it is amongst the most relevant for learning. [...] The joint treatment of patients in the emergency ward with experienced colleagues was the most instructive of the things I've had to do since my exams. [07].

- In between there are a few interesting cases for which one just needs more help and for which one consults someone, someone experienced. In fact, one learns most this way. [01]

Consults facilitate a broad spectrum of medical and clinical knowledge and skills. They contain and subsume a number of practices relevant to learning for on-call as well as for requesting doctors.

- One can learn: systematic patient examination, anamnesis, decision-making, therapy: options and alternatives. [01]

- It is definitely relevant because it brings together everything one does throughout the day. From interpreting laboratory values and images through anamnesis and examination and finally decision making. Everything we do is part of it. [04]

The specialist knowledge involved appeared to be fascinating for requesting doctors. In particular inexperienced doctors deemed the opportunity to interact with specialists to be valuable and highlighted its relevance for learning.

- It [the knowledge of the consultant is specialised knowledge which means it mostly is detailed and therefore fascinating. [09]

- I find it very exciting because you talk to the specialists in the field. I am still a blank canvas. I still have little idea about surgery or orthopaedics as I started here only recently. That is why I learn a lot. [06]

Some particularly appreciated consults with experts from specialisms or sub-specialisms close to their intended specialisation. These were of greater interest and tended to lead to a more intensive engagement, for example in the form of questions to the on-call doctors.

- $[\ldots]$ more in the areas in which one has developed further. They tended to interest me more and one is more likely to probe further. [05]

Also on-call doctors considered consults as unique learning opportunities. While they usually exercised less responsibility in their departments, in making or confirming a diagnosis in the context of a consult they had to act as frontline 'experts':

- In terms of patient care in the context of consults one is in the frontline. Particularly as resident, because one is on-call, has to think and show presence. In such situations one is initially more involved in the process compared with patients having been admitted for stationary treatment already and been treated or operated on by more experienced colleagues, senior doctors or head doctors. [08] 
- I consider it very relevant for learning because one is being called to consult as someone who works here but with limited experience. [07]

Both residents in the role of on-call and requesting doctors valued the feedback they received in consults. They found it particularly helpful that they received feedback upon their own problem-solving processes that allowed them to contrast their own practices and concepts with the approaches of experts. They deemed this a unique learning opportunity provided in the context of consults.

- Of special significance is that one has already seen the patient and that one has already formed an opinion before the specialist arrives. It is as if you get a corrected exam back. [04]

While also senior doctors stated that they can learn from consults, they were considered to be most relevant for the learning of residents.

- I think that everyone can benefit. Ideally it is important for the resident doctor who is still in training. [02]

Cognitive apprenticeship methods in doctor-to-doctor consultation

RQ2 How do the pedagogical approaches associated with CA facilitate learning in interdisciplinary cooperation such as consults?

In the next step, we analysed if the value of consults for learning can also be confirmed from the perspective of CA, which is widely considered to be a useful instructional paradigm when experts need to explain complex tasks to learners (Collins et al. 1991). Consequently, we took the 'methods' of CA as an analytical framework. We individually examined how each 'method' was applied in the context of consults in terms of frequency, form and quality.

\section{Modelling}

In modelling learners observed experts who were performing and explaining tasks. While it is acknowledged that physical activities - which also embody important cognitive skills (Brown et al. 1989) — can to some extent be learned through observation, in non-physical domains experts need to externalize their mental processes more explicitly (Collins et al. 1991). All participants reported the latter form of modelling, as requesting doctors always received the advice of on-call doctors. ED residents recognised the value of modelling for their learning and also on-call doctors confirmed that point of view. There was, however, much variance with respect to the quality of the feedback of modelling: the range was from short text messages to detailed and rich oral explanations and sketches of the reasons underlying problem solving. The latter was deemed to be very relevant for learning.

- Sometimes it is very instructive when an orthopaedist explains what sort of fracture is in evidence and how to treat it. It is instructive because one can develop the confidence to have a go oneself. Sometimes it is very informal: 'Do this and this.' This is the range of possibilities. [03] 
Oral explanations that were accompanied by physical demonstration directly at the bedside were also highly appreciated. In the data there was comparatively little evidence for that form of bedside teaching. While residents both in the role of requesting and as oncall doctors definitely appreciated active forms of modelling as learners, they indicated that they benefited even without explicit teaching by experts, by simply listening to and observing the experts.

- I also learned during examinations. It is fascinating how they approach a clinical examination. [10]

The extent to which modelling can take place was significantly determined by workload and the availability of time. First of all, requesting doctors in the ED needed to have the time to take part in the examination of the patient by the on-call doctors. In emergency departments which account for a large number of cases and which are characterised by overcrowding (Derlet et al. 2001), it cannot be taken for granted, as one doctor explained: He [on-call doctor] then goes to the patient. I mostly try to come along if I have the time to do so. [04]. The quality of modelling, i.e. the extent to which the on-call doctors make their thinking explicit, is also strongly dependent on workload.

\section{Coaching}

Coaching is described as observing learners while they carry out tasks and offering advice (Collins et al. 1991). In the domain of physical tasks we hardly found any evidence off coaching. If the requesting doctor took part in the physical examination of the patient, in most cases the more experienced (or specialist) doctor led on the patient treatment. This facilitated learning by modelling which we discuss above. "He [the on-call doctor] introduces himself and depending on the case he carries out an examination." [09]. Considerably more often doctors received feedback and advice when they articulated the case via telephone or in situ and suggested a treatment. This held particularly true for oncall doctors when they involved their attendings and learned in an intradisciplinary setting.

- And a second learning effect is that I also frequently phone the attending doctor in the background and he gives me additional feedback as to whether he agrees or if an alternative treatment is advisable. For me, as the person being called to the consultation, it is very instructive. [07]

\section{Scaffolding}

Scaffolding is the interactional and meditational support the teacher provides to the student. In the context of consults, scaffolding was triggered by the learner. This allowed for scaffolding in loosely-coupled, interdisciplinary cooperation between different departments. In this sense, scaffolding can be considered as an inherent part of a consult: requesting doctors triggered scaffolding in case they felt that they were not capable to manage the situation on their own. In involving (sub-)specialties on demand, consults were described as practices where the requesting doctor had to decide on when to use scaffolding that, again, can could to modelling. 
- I seek consultation if I don't know how to proceed or if I am not sure what I should do exactly. [04]

Subsequently, the form of scaffolding was decided on, for example, if there was a need for 'on-site scaffolding' or if the requesting doctor was able to be supported via telephone. In most cases of interdisciplinary consultation, the on-call doctors needed to see and examine the patient personally. The same pattern was true for an on-call resident when involving her/his attending. Also, scaffolding was provided in the form of telephone consultation or through on-site support. More telephone-based scaffolding was involved in this form of intradisciplinary cooperation. According to Collins et al., scaffolding is closely linked to fading which means "the gradual removal of supports" (1991). There was much evidence that successful scaffolding prepared the learner for similar cases in the future and, that more experienced doctors involved the specialists less often.

- In the process one learns, one considers a possible treatment, makes the call, explains the case and proposes a course of action. Then one gets the go-ahead and one knows by implication whether one has deliberated correctly. Or they give further advice for future reference. That way one can optimise everything. [08]

\section{Articulation}

Articulation involves any method of getting students to articulate their knowledge, reasoning, or problem solving processes (Collins et al. 1991). Some on-call attendings reported that they actively and deliberately tried to get residents to articulate their concepts and to make suggestions for further procedures in order to support their learning within a consult. The example below shows that articulation was not limited to members of the respective discipline but can also include ED residents:

- I also always try to elicit therapy suggestions from the resident, ascertain what he would do, what treatment he would recommend. [...] That way he can develop his own approach for dealing with such problems. [...] So one would say 'the patient has a flexor tendon injury' and then one asks the resident from the ED how he would treat such an injury. [...] We would ask our own residents more searching questions. [05]

However, even without deliberate teaching intentions the consult required requesting doctors to communicate their view on the problem and possible problem-solving strategies over the phone to the on-call doctor. This was also the case for on-call residents who involved their attendings and articulated the case.

- The caller says where the call comes from, name and date of birth of the patient, why he is hospitalised, what happened, why a particular specialist is needed and what he thinks the problem is. [04]

\section{Reflection}

According to CA, student reflection is stimulated by comparing "their own problemsolving processes with those of an expert, (or) another student" (Collins et al. 1991). This 
is also part of consults, where learners have the opportunity to observe experts' procedures (in the sense of modelling) and compare them to their previous approaches. This is also the case in the acquisition of non-physical skills, where learners can compare their cognitive concept to the solution favoured by the expert. We found evidence that in consults learning from reflection by both requesting doctors and on-call doctors was very much triggered by potential errors where their ideas and mental concepts of how to solve the problem differed considerably from that of the experts.

- This is exactly the engramme which one prepares oneself. Then, the expert tells you otherwise. This means one's trail of thought was erroneous. One remembers that. [06]

In consults there exist no techniques to 'replay' the performance of both expert and novice for comparison as favoured by Collins et al. (1991). However, doctors engaged in post hoc reflection by discussing their experiences with colleagues and by reading case-related material.

- If one discusses a case again and if one reads after the discussion through the symptoms or the injury patterns again in the textbook, one can make a mental note of it. [08]

\section{Exploration}

CA understands exploration as pushing students into a mode of problem solving on their own. It includes fading in problem solving and fading in problem setting (Collins et al. 1991). In the context of consults both requesting doctors and on-call doctors were pushed into problem solving. To a certain extent they needed to treat patients independently and take decisions on their own. Sometimes on-call doctors decided on (the degree of) exploration in that they determined whether the requesting doctors can treat the patients on their own:

- $\quad[\ldots]$ then the on-call doctor comes by and looks at the patient. According to the situation he tells us to treat on our own. [06]

The degree to which residents took responsibility and engaged in exploratory learning, however, was only guided by experts to some extent. It also depended on the interplay of a number of organisational and situational factors such as role, size and scope of the hospital. As we have already indicated, the role of an on-call doctor (and not the explicit instruction of an expert) allowed a high degree of learning by exploration in that, for example, a relatively inexperienced on-call doctor independently saw and examined patients. Also, the role of ED residents, who acted as requesting doctors, included much exploration, particularly in smaller hospitals. There, due to the limited availability of specialties, residents engaged very much in learning by exploration. In addition, the extent of exploratory learning also depended on the organisation of shifts. During night shifts there is a limited availability of experts in many hospitals. And finally, exploratory learning, in particular for relatively inexperienced doctors, was not only common in consults, but also deemed to be highly relevant by both requesting doctors and on-call doctors for their own learning. 
- At the moment in most instances I decide what the patient needs and what the treatment is. I initiate things. [...] If you do it the first time, that means a very different degree of pressure and concentration. One learns a lot like that. [01]

In the context of consults we did not, however, find an explicit setting of general learning goals and no encouragement to focus on sub-goals of particular interest by the teacher, as suggested by CA to support and guide exploration (Collins et al. 1991).

\section{Discussion}

In the following we discuss the results from various theoretical perspectives. First, we contrast our findings with related studies on medical and clinical problem solving. Then we introduce a model that we developed to better illustrate the interplay of pedagogical features of CA in the context of collaborative and interdisciplinary problem-solving. This is followed by a critical discussion of the CA in the context of interdisciplinary cooperation. Finally, we analyse and discuss influences on the 'methods' of CA other than deliberate, teacher-driven actions such as contextual factors.

\section{Perceived value of inter- and intradisciplinary problem solving for learning}

There is much evidence in the data that cooperation of doctors across disciplines in the form of consults is not only a common and frequent phenomenon of contemporary patient care, but is central to medical and clinical competence development. We showed how complex medical problems, which are treated across departmental boundaries can trigger rich forms not only of interdisciplinary but also of intradisciplinary learning. This is particularly the case for residents who work in hospitals as part of their educational curriculum and often act as requesting doctors as well as on-call doctors. Doctors in both roles can learn in or from consults. The study reinforces the importance of problem solving and 'breakdowns' for learning. The findings can, for example, be related to Slotnick's conceptualisation in that the process of a consult is considered as learning linked to specific problems which takes place ad-hoc and relates to a specific need. Smith et al. (2004) also describe "effective responses to breakdowns" that were closely associated with reflexive learning. Therefore, consults might be viewed as effective responses with relevance for learning because the resident encounters a problem which s/he can solve with the help of a more experienced doctor. In this way, as we have shown, a number of activities relevant for learning can be triggered. In addition, the relevance of 'near-miss errors' in the context of consults becomes evident: doctors describe how they learn in doctor-to-doctor consultation through mistakes they nearly made had it not been for the consultation of a more specialised/experienced doctor.

\section{Interplay of pedagogical methods of CA}

Whilst we have not so far explored social interaction, i.e. practices of relevance for teaching and learning with experienced colleagues, in any detail, we were able to show with references to CA how doctors learn from a socio-cognitive perspective in the context of consults. Experts can-in their role as teachers-stimulate learning practices by using the pedagogical approaches of CA within the context of consults. They can provide opportunities for modelling, reflection and scaffolding, engage in coaching, get the learners 
to articulate their knowledge and push them to problem solve. This appears to take place according to certain patterns, which we will discuss in the following sections (see also Fig. 1).

Typically, CA does not begin with modelling but with exploration: residents (requesting doctors as well as on-call doctors) first of all have to master a task by themselves. If they encounter a problem, they are required to articulate it together with possible solutions to an experienced and/or specialist colleague (articulation). This way they trigger different types of scaffolding in the form of modelling and/or coaching: if a doctor involves the on-call doctor, the latter normally comes to ED and examines the patient. She or he cannot only make decisions, but through modelling explicate underlying considerations as well as demonstrate practices of diagnosis and treatment in relation to the patient. If the on-call resident involves her/his attending over the telephone, s/he also articulates a possible treatment solution and is being coached through suggestions, hints and feedback. Then, the attending on-call can also come to the ED and can enable learning by modelling in situ. And although on-call doctors tend to involve their attendings more over the telephone that through on-site modelling, it becomes clear that the interaction patterns around help seeking by residents in the ED and on-call doctors involving their attendings are similar. Reflective learning in CA terms is triggered in that residents compare their treatment solutions with those of experienced (specialist) doctors and receive targeted feedback (reflection).

The application of methods is not necessarily linear but can be iterative. Articulation, for example, can recur during the examination of the patient, after coaching on the telephone. Modelling can follow on from coaching, for example, if the doctor offering advice does so in the form of coaching tips and feedback over the telephone, and subsequently in the ED through direct observation; the same applies to the on-call resident who involves his/her attending. This demonstrates how much approaches to and quality of learning are dependent on clinical processes.

Critical appraisal of CA in the context of interdisciplinary cooperation

In taking a critical look at CA as a tool for analysis, one needs to note that the teaching methods are interlinked and cannot be easily separated: Collins et al. consider coaching as "observing students while they carry out a task and offering hints" (Collins et al. 1991);

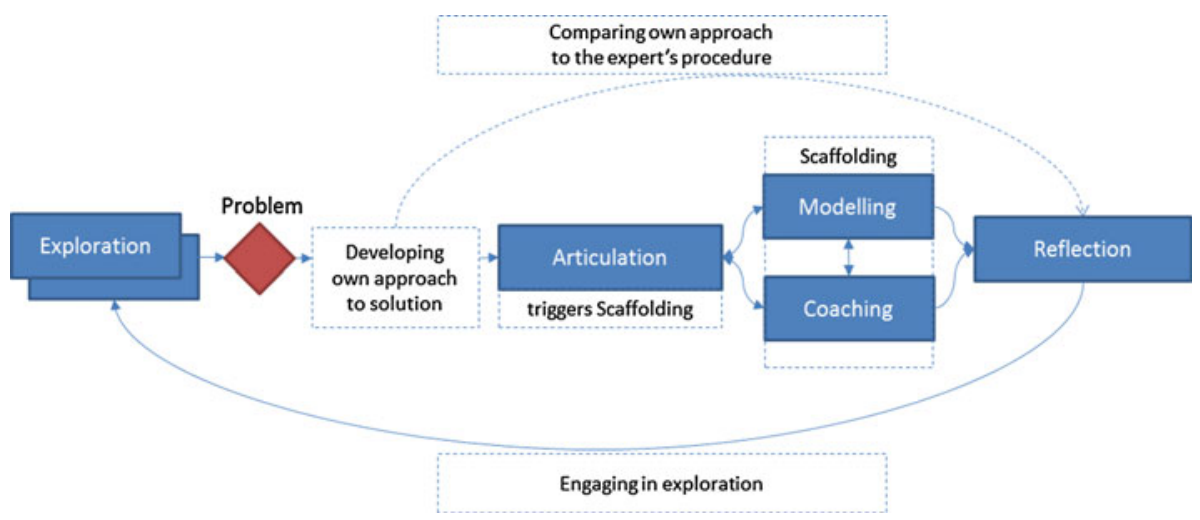

Fig. 1 Model of CA methods in doctor-to-doctor consultations 
similarly, they describe scaffolding as "the supports the teacher provides to help the student carry out the task. These supports can take either the forms of suggestions or help" (Collins et al. 1991). Consequently, in the model above coaching is depicted as a form of scaffolding and (even) modelling can be considered as scaffolding if it is triggered in a problem-solving situation. In addition, the methods are tightly linked and connected as the following example shows: if a learner articulates a solution to a problem and receives feedback, this can, in turn, be considered as coaching. These similarities and interrelationships make a clear and disjunctive categorisation difficult. This does not, however, limit the value of CA as an approach that can guide teachers - or experts - and helps them to engage in relevant teaching practices. Accordingly, the value of CA is not restricted to formal learning settings with more or less explicit learner-teacher relations such as schools. CA can also help experts/teachers to stimulate more explicitly student learning in informal learning contexts such as problem solving and cooperation in clinical settings.

An explorative approach to learning and teaching, with which CA could be expanded, is for less experienced doctors in a specialist discipline to assume the role of experts. Collins et al. (1991) considered "reciprocal teaching', where teacher and students take turns playing the role of teacher," as very effective in context of reading acquisition. Interdisciplinary cooperation in the form of consults goes beyond that in so far as the coach is no longer present and the learners (residents on-call) have to assume the role of an expert in relation to third parties, which tends to be instructive and motivating. In this context the availability of an expert, who can assist in case of uncertainties, is essential.

CA methods: teacher- or context-driven?

The findings also show that, similarly to results reported by van de Wiel et al. (2010), learning and teaching in general and in particular the application of the six 'methods' of CA only partly take place deliberately. Accordingly, much could be gained, we would argue, by managing learning and teaching opportunities more explicitly. In order to leverage consults for learning, experienced and specialist doctors have to be aware of its huge potential and explain the underlying reasons behind their problem solving. In this sense, externalisation of knowledge or-in CA terms- 'making thinking visible', is central to learning by solving problems in doctor-to-doctor consultations.

However, beyond deliberate actions of experts or teachers, learning and teaching practices are also very much driven by the consultation process per se and are influenced by other situational and organisational variables: for example, modelling can be considered an inherent part of consults, as the process per se requires feedback and explanations from the on-call doctor to the requesting doctor. We also found evidence that time and workload and the organisation of shifts impact on, for example, whether bedside teaching and modelling actually take place, i.e. whether the requesting doctor can accompany the on-call doctor during the patient examination, or whether the attending of the latter can explain and 'model' in situ; and, to what extent the actors involved in consultations make their thinking explicit to each other. Also, scaffolding and coaching are more often triggered by the learner in case of insecurities and questions in the process of treating a patient than by deliberate teaching intentions. Furthermore, we found many examples for process-driven articulation in consults. In these contexts, articulation can be considered more as a necessity of inter- and intradisciplinary cooperation processes than a deliberate articulation method applied by experts to stimulate residents' learning. Requesting doctors, for example, have to articulate relevant case details as well as their perspectives on the problem over the phone in order to inform the on-call doctor at the beginning of a consult. 
Reflection appears also to be very much stimulated by the responsibility and the role that relatively inexperienced on-call doctors have to take in consults as well as by "potential errors". Similarly, the extent to which residents learn through exploration seems to depend on situational and organisational factors such as role and responsibility as well as on other factors such as size and scope of the hospital.

\section{Limitations and outlook}

We consider consults as typical and suitable practices to explore learning from the perspective of interdisciplinary co-operation and problem solving. It has to be acknowledged, however, that they represent just one possible form of interdisciplinary work. Whilst we discussed here in the main learning and teaching practices in inter- and intradisciplinary cooperation, we explored significance and interplay of situational, organisational and procedural factors rather selectively. With respect to methodology our research was exclusively based on interviews, and therefore on self-reported and perceptual data, and did not use any other form of qualitative or quantitative data collection.

While we considered all roles which are typically involved in emergency consults and while we conducted research in two hospitals varying in scope and size, we involved a rather small number of interview partners from two cultural settings. These limitations might be considered as sample bias und must be taken into account when generalising the findings for other contexts. From a theoretical perspective, CA is a broad and pragmatic frame which integrates a number of recognised approaches to teaching and learning. It should be noted, however, that the same approaches are discussed in a more differentiated and profound way in (research) literature not associated with CA. For example, there exists a significant body of literature on mentoring and coaching across a range of disciplines; the notion of scaffolding was discussed by the cognitive psychologist Jerome Bruner et al. (see, for example, Wood et al. 1976) drawing on the work of Lev Vygotsky; or, Schön $(1983,1987)$ developed a sophisticated concept of reflection in relation to work practices. A detailed theoretical engagement with these pedagogical approaches, though desirable, would go well beyond the scope of this paper.

In view of the focus and thematic limitations of this paper we encourage future research to consider learning that may arise from other forms of interdisciplinary and interprofessional work such as interactions in operating theatres, less formal co-operation between actors of different disciplines or professions, and consults between departments that are not centred on emergency wards. We also propose that further research should expand the geographical and cultural scope. From a methodological perspective we would suggest participatory observations and ethnological approaches so as to contrast and triangulate the perceptual data with observational data. As indicated, we were able to explore the significance of situational, organisational and procedural factors rather selectively. Accordingly (and as we would argue most importantly), future research should explore these correlations more in-depth. Therefore, we would suggest theoretical triangulation by using other socio-cognitive and situated approaches such as distributed cognition or ecological theories in order to better explore the interplay of contextual factors and learning (see, for example, Durning et al. 2010; Nardi 1996). In addition, we would propose using grounded theory and approaches that develop theory (rather) inductively from a corpus of data in order to identify themes and particularities of the clinical context not covered by existing theoretical concepts. 


\section{Conclusion}

Against the background of increasing specialisation in the field of healthcare and the resultant necessity for interdisciplinary cooperation, we analysed in this paper how, and to what extent the cooperation between hospital doctors across departmental boundaries impacts on their learning and competence development. We have shown how more complex patient cases, which are treated in the context of consults, facilitate rich forms not only of interdisciplinary but also of intradisciplinary learning. On the one hand the doctors participating in the study considered cooperation in the context of a consult as one of the most relevant clinical activities for learning as it presents numerous and varied opportunities for learning both for the requesting doctor as well as the on-call doctor in particular if residents occupy both these roles. The findings confirm existing studies which consider the solving of problems in everyday clinical practice as very relevant for learning. Research to date has tended to focus on individual and cognitive-psychological aspects of clinical problem solving and has only sporadically noted the importance of social interaction and attendant teaching and learning practices in the context of problem solving. The present study has, therefore, attempted to combine social and cognitive perspectives with reference to cognitive apprenticeship. The results show how experienced doctors assume a teaching role making use of the pedagogical approaches of CA, partly deliberate, but in varying form, degree of frequency and quality and thereby stimulate learning in other, less experienced doctors. Accordingly, CA may be able to assist experts also in fostering the learning of less experienced colleagues in informal learning contexts such as a clinical setting more deliberately. Whilst we discussed here in the main learning and teaching practices in inter- and intradisciplinary collaboration and whilst we could only explore the significance of situational, organisational and procedural factors rather selectively future research should explore these correlations in more depth.

Acknowledgments We thank our project sponsors and partners, CTI-the Swiss Confederation's Innovation Promotion Agency, AMTS, Agfa Healthcare, University Hospital Basel, Hightech Research Center of Cranio-Maxillofacial Surgery University of Basel and the University of Applied Sciences Northwestern Switzerland for their support of this study.

\section{Appendix: Interview guide}

\section{Consults: processes/characteristics/interactions}

- How do you experience consults? Please explain a typical consultation process as detailed as possible/step-by step

If not already addressed, the following sub-questions are discussed:

- How and why/in which situations is a consult initiated? Please provide examples.

- Who/what role/s is/are usually involved in consults?

- What are responsibilities attached to the roles?

- Are there different forms of consults? Is there variation in how a consult takes place?

- How often are you involved in consults?

- How do you interact with doctors in a consult? Please provide examples.

- What tools/media are involved in consults? 
Perceived learning and consults

- What is you understanding of learning?

- How do you learn in/through your clinical practice? Please provide examples.

- Do you learn something in/through consults?

- How do you learn in consults? Please provide concrete examples/situations.

- Do you think other actors involved in consults can learn?

- How do other actors involved in consults learn? Please provide concrete examples/ situations.

- What do you learn in/through consults? What sort of knowledge/skills/competences etc. do you gain in consults? Please provide examples.

- With respect to learning: are consults more (or less) relevant compared to other clinical activities? Why? What are particularities of consults?

- In which situations do you learn best in consults? Please provide examples.

- With/from whom do you learn in consults?

\section{References}

Balmer, D. F., Serwint, J. R., Ruzek, S. B., \& Giardino, A. P. (2008). Understanding paediatric residentscontinuity preceptor relationships through the lens of apprenticeship learning. Medical Education, 42(9), 923-929.

Bardram, J. E., \& Bossen, C. (2005). Mobility work: The spatial dimension of collaboration at a hospital. Computer Supported Cooperative Work (CSCW), 14(2), 131-160.

Barr, H., Koppel, I., Reeves, S., \& Hammick, M. (2005). Effective interprofessional education: Argument, assumption, and evidence. Oxford: Blackwell.

Baxter, P., \& Jack, S. (2008). Qualitative case study methodology: Study design and implementation for novice researchers. The Qualitative Report, 13(4), 544-559.

Becker, J., Bergener, K., Mueller, O., \& Mueller-Wienbergen, F. (2009). Documentation of flexible business processes-A healthcare case study. Paper presented at the Americas Conference on Information Systems, San Francisco.

Bennett, N. L., Casebeer, L. L., Zheng, S., \& Kristofco, R. (2006). Information-seeking behaviors and reflective practice. Journal of Continuing Education in the Health Professions, 26(2), 120-127.

Bleakley, A. (2006). Broadening conceptions of learning in medical education: The message from team working. Medical Education, 40(2), 150-157.

Brown, J. S., Collins, A., \& Duguid, P. (1989). Situated cognition and the culture of learning. Educational Researcher, 18(1), 32-42.

Carpenter, J. (1995). Interprofessional education for medical and nursing students: Evaluation of a programme. Medical Education, 29(4), 265-272.

Collins, A., Brown, J. S., \& Holum, A. (1991). Cognitive apprenticeship: Making thinking visible. American Educator, 15(3), 6-11.

Collins, A., Brown, J. S., \& Newman, S. E. (1987). Cognitive apprenticeship: Teaching the craft of reading, writing, and mathematics. Cambridge, MA: lllinois University (Urbana: Center for the Study of Reading. Bolt, Beranek and Newman, Inc).

Cortazzo, J. M., Guertler, A. T., \& Rice, M. M. (1993). Consultation and referral patterns from a teaching hospital emergency department. The American Journal of Emergency Medicine, 11(5), 456-459.

Covell, D. G., Uman, G. C., \& Manning, P. R. (1985). Information needs in office practice: Are they being met? Annals of Internal Medicine, 103, 596-599.

Derlet, R. W., Richards, J. R., \& Kravitz, R. L. (2001). Frequent overcrowding in US emergency departments. Academic Emergency Medicine, 8(2), 151-155.

Dornan, T., Boshuizen, H., King, N., \& Scherpbier, A. (2007). Experience-based learning: A model linking the processes and outcomes of medical students' workplace learning. Medical Education, 41(1), 84-91.

Durning, S. J., Artino, A. R., Jr, Pangaro, L. N., Van Der Vleuten, C., \& Schuwirth, L. (2010). Perspective: Redefining context in the clinical encounter: Implications for research and training in medical education. Academic Medicine, 85(5), 894-901. 
Eisenhardt, K. M. (1989). Building theories from case study research. The Academy of Management Review, 14(4), 532-550.

Ericsson, K. A. (2004). Deliberate practice and the acquisition and maintenance of expert performance in medicine and related domains. Academic Medicine, 79(10), S70.

Ericsson, A. (2008). Deliberate practice and acquisition of expert performance: A general overview. Academic Emergency Medicine, 15(11), 988-994.

Fagin, C. M. (1992). Collaboration between nurses and physicians: No longer a choice. Academic Medicine, 67(5), 295.

Furmedge, D. S. (2008). Apprenticeship learning models in residents. Are they transferable to medical students? Medical Education, 42, 856-857.

Gemmel, P., Vandaele, D., \& Tambeur, W. (2008). Hospital Process Orientation (HPO): The development of a measurement tool. Total Quality Management \& Business Excellence, 19(12), 1207-1217.

Giacomini, M. K., \& Cook, D. J. (2000). Users' guides to the medical literature. Qualitative research in health care. Are the results of the study valid? JAMA: The Journal of the American Medical Association, 284(3), 357-362.

Go, S., Richards, D. M., \& Watson, W. A. (1998). Enhancing medical student consultation request skills in an academic emergency department. Journal of Emergency Medicine, 16(4), 659-662.

Goldman, L., Lee, T., \& Rudd, P. (1983). Ten commandments for effective consultations. Archives of Internal Medicine, 143(9), 1753.

Hall, P., \& Weaver, L. (2001). Interdisciplinary education and teamwork: A long and winding road. Medical Education, 35(9), 867-875.

Kirschner, P. A. (2006). (Interdependent) Learning. Learning is interaction. Inaugural address. Utrecht: Cip-gegevens Koninklijke Bibliotheek, Den Haag.

Lavin, M., Ruebling, I., Banks, R., Block, L., Counte, M., Furman, G., et al. (2001). Interdisciplinary health professional education: A historical review. Advances in Health Sciences Education, 6(1), $25-47$.

Lee, R. S., Woods, R., Bullard, M., Holroyd, B. R., \& Rowe, B. H. (2008). Consultations in the emergency department: A systematic review of the literature. Emergency Medicine Journal, 25(1), 4.

Mayring, P. (2004). Qualitative content anaiysis. In U. Flick, E. von Kardorff, \& I. Steinke (Eds.), A companion to qualitative research (pp. 266-269). London, Thousand Oaks (New Delhi: SAGE Publications Ltd).

Merriam, S. (1998). Qualitative research and case study applications in education. Revised and expanded from "case study research in education". San Francisco: Jossey-Bass Publishers.

Nardi, B. A. (1996). Studying context: A comparison of activity theory, situated action models, and distributed cognition. In B. A. Nardi (Ed.), Context and consciousness: Activity theory and humancomputer interaction (pp. 69-102). Massachusetts Institute of Technology.

Parsell, G., \& Bligh, J. (1998). Interprofessional learning. Postgraduate Medical Journal, 74(868), 89.

Patel, V., Yoskowitz, N., \& Arocha, J. (2008). Towards effective evaluation and reform in medical education: A cognitive and learning sciences perspective. Advances in Health Sciences Education, 14(5), 791-812.

Patton, M. Q. (2002). Qualitative research and evaluation methods (Vol. 3). Thousand Oaks: Sage Publications, Inc.

Salerno, S. M., Hurst, F. P., Halvorson, S., \& Mercado, D. L. (2007). Principles of effective consultation: An update for the 21st-century consultant. Archives of Internal Medicine, 167(3), 271.

Sargeant, J., Mann, K., Sinclair, D., Ferrier, S., Muirhead, P., van der Vleuten, C., et al. (2006). Learning in practice: Experiences and perceptions of high-scoring physicians. Academic Medicine, 81(7), 655-660.

Schön, D. A. (1983). The reflective practitioner-How professionals think in action. New York: Basic Books.

Schön, D. A. (1987). Educating the reflective practitioner. Toward a new design for teaching and learning in the professions. San Francisco: Jossey-Bass.

Slotnick, H. B. (1999). How doctors learn: Physicians' self-directed learning episodes. Academic Medicine, 74(10), 1106.

Smith, C. S., Morris, M., Francovich, C., Hill, W., \& Gieselman, J. (2004). A qualitative study of resident learning in ambulatory clinic. The importance of exposure to 'breakdown' in settings that support effective response. Advances in Health Sciences Education, 9, 93-105.

Stalmeijer, R., Dolmans, D., Wolfhagen, I., \& Scherpbier, A. (2008). Cognitive apprenticeship in clinical practice: Can it stimulate learning in the opinion of students? Advances in Health Sciences Education, 14(4), 535-546.

Sternas, K. A., O’Hare, P., Lehman, K., \& Milligan, R. (1999). Nursing and medical student teaming for service learning in partnership with the community: An emerging holistic model for interdisciplinary education and practice. Holistic Nursing Practice, 13(2), 66. 
van de Wiel, M. W. J., Van den Bossche, P., Janssen, S., \& Jossberger, H. (2010). Exploring deliberate practice in medicine: How do physicians learn in the workplace? Advances in Health Sciences Education, 16(1), 81-95.

Vogel, D. (1993). Patient-focused care. American Journal of Health-System Pharmacy, 50(11), 2321-2329.

Vosk, A. (1998). Response of consultants to the emergency department: A preliminary report. Annals of Emergency Medicine, 32(5), 574-577.

Wood, D., Bruner, J. S., \& Ross, G. (1976). The role of tutoring in problem solving. Journal of Child Psychology and Psychiatry, 17(2), 89-100.

Woods, R. A., Lee, R., Ospina, M. B., Blitz, S., Lari, H., Bullard, M. J., et al. (2008). Consultation outcomes in the emergency department: Exploring rates and complexity. CJEM: Canadian Journal of Emergency Medical Care, 10(1), 25.

Woolley, N., \& Jarvis, Y. (2007). Situated cognition and cognitive apprenticeship: A model for teaching and learning clinical skills in a technologically rich and authentic learning environment. Nurse Education Today, 27(1), 73-79. 